\title{
Distinct Pathways for the Early Recruitment of Myosin II and Actin to the Cytokinetic Furrow
}

\author{
Mian Zhou and Yu-Li Wang \\ University of Massachusetts Medical School, Department of Physiology, Worcester, MA 01605
}

Submitted August 14, 2007; Revised September 21, 2007; Accepted October 17, 2007

Monitoring Editor: Fred Chang

Equatorial organization of myosin II and actin has been recognized as a universal event in cytokinesis of animal cells. Current models for the formation of equatorial cortex favor either directional cortical transport toward the equator or localized de novo assembly. However, this process has never been analyzed directly in dividing mammalian cells at a high resolution. Here we applied total internal reflection fluorescence microscope (TIRF-M), coupled with spatial temporal image correlation spectroscopy (STICS) and a new analytical approach termed temporal differential microscopy (TDM), to image the dynamics of myosin II and actin during the assembly of equatorial cortex. Our results indicated distinct and at least partially independent mechanisms for the early equatorial recruitment of myosin and actin filaments. Cortical myosin showed no detectable directional flow during early cytokinesis. In addition to equatorial assembly, we showed that localized inhibition of disassembly contributed to the formation of the equatorial myosin band. In contrast to myosin, actin filaments underwent a striking flux toward the equator. Myosin motor activity was required for the actin flux, but not for actin concentration in the furrow, suggesting that there was a flux-independent, de novo mechanism for actin recruitment along the equator. Our results indicate that cytokinesis involves signals that regulate both assembly and disassembly activities and argue against mechanisms that are coupled to global cortical movements.

\section{INTRODUCTION}

Recruitment of myosin II (referred as myosin later) and actin along the equatorial cortex represents a universal event in cytokinesis. Although the function of a narrowly defined "contractile ring" is still under debate (Wang, 2005), it is generally agreed that the organization of actin and myosin filaments along the equator is related to equatorial contractility and cortical ingression. There are two prevailing hypotheses on how myosin and actin are recruited to the equatorial cortex. The cortical flow hypothesis proposes that myosin and actin from the polar cortex flow actively or passively into the equatorial cortex, as a consequence of either directed transport or differential cortical contractions (White and Borisy, 1983; Bray and White, 1988). The structural synthesis hypothesis argues that direct recruitment of molecules or small polymeric building blocks from the cytoplasm is responsible for the formation of the acto-myosin equatorial band (Pelham and Chang, 2001; Wu et al., 2006).

There is experimental evidence for the involvement of both cortical flow and direct structural synthesis in cytokinesis. The former was supported primarily by imaging membrane-bound beads (Wang et al., 1994), fluorescently labeled actin (Cao and Wang, 1990) or myosin (DeBiasio et al., 1996) in systems from live mammalian cells to Dictyostelium. In addition, several theoretical models predicted a concerted flow of cortical actin and myosin into the equator (White and Borisy, 1983; He and Dembo, 1997), as a consequence of global force balance over the cortex. Direct structural synthesis was supported by the appearance and/or

This article was published online ahead of print in $M B C$ in Press (http:/ / www.molbiolcell.org/cgi/doi/10.1091/mbc.E07-08-0783) on October 24, 2007.

Address correspondence to: Yu-Li Wang (yuli.wang@umassmed.edu). growth of punctate acto-myosin structures in dividing frog embryos (Noguchi and Mabuchi, 2001), and yeast (Wu et al., 2006) and by the dependence of cytokinesis on genes that regulate de novo structural assembly (Gunsalus et al., 1995; Pelham and Chang, 2001; Severson et al., 2002). Although cortical flow and structural synthesis hypotheses are not mutually exclusive, their contributions to the recruitment of equatorial actin and myosin remain unclear. As several hypothetical mechanisms of cytokinesis make strong predictions about how equatorial structures are formed (Burgess and Chang, 2005), detailed analyses of cortical dynamics during early cytokinesis are likely to provide useful new insights.

The limitation to the current knowledge is in part due to the lack of high-resolution images that depict the assembly of equatorial cortex immediately before and during cytokinesis. Because of the strong signals from the cytoplasm, it has been very difficult to observe directly the assembly process in live cells using conventional epifluorescence optics. With its very short depth of field $(<200 \mathrm{~nm})$ and dramatically reduced background, the total internal reflection fluorescence microscopy (TIRF-M) is uniquely suitable for providing high-resolution images of structural dynamics on the cell cortex (Axelrod, 2001). In this study, we have combined TIRF-M with spatial temporal image correlation spectroscopy (STICS; Hebert et al., 2005), an approach similar to fluorescence speckle microscopy for precise tracking of the movement of structural inhomogeneities, and temporal differential microscopy (TDM), a novel image analysis method that measures the local kinetics of structural assembly or disassembly. Our observations lead us to the conclusion that actin and myosin follow distinct pathways to the equatorial cortex. Cortical flow is limited to actin and is not solely responsible for its equatorial concentration, whereas myosin is recruited directly from the cytoplasm. In addition, we 
show that the formation of equatorial myosin band involves the regulation of both assembly and disassembly activities.

\section{MATERIALS AND METHODS}

\section{Cell Culture and Transfection}

A subclone of normal rat kidney cells (NRK-52E; American Type Culture Collection, Rockville, MD) was grown on glass coverslips in Kaighan's modified F12 medium (Sigma, St. Louis, MO) supplemented with $10 \%$ fetal calf serum (JRH Biosciences, Kansas City, MO), $1 \mathrm{mM}$ L-glutamine, $50 \mu \mathrm{g} / \mathrm{ml}$ streptomycin, and $50 \mathrm{U} / \mathrm{ml}$ penicillin (Invitrogen, Carlsbad, CA). Cells were maintained at $37^{\circ} \mathrm{C}$ in a humidified atmosphere containing $5 \% \mathrm{CO}_{2}$. Enhanced green fluorescent protein (EGFP)-nonmuscle myosin heavy chain IIA and IIB, EGFP-actin, and mCherry-actin plasmids were kindly provided by Dr. Robert S. Adelstein (National Institutes of Health, National Heart, Lung, and Blood Institute) and Dr. Michael Davidson (Florida State University). Transient transfection of NRK epithelial cells was performed by nucleofection using the Amaxa nucleofector and kit R (Amaxa, Gaithersburg, MD), following the manufacturer's protocol. After transfection, cells were plated on glass coverslips and cultured overnight before imaging.

\section{Drug Treatment and Microinjection}

The drug concentrations of Y-27632 (Calbiochem, San Diego, CA), (-)-blebbistatin (Toronto Research, Toronto, ON, Canada), (+)-blebbistatin (Calbiochem, San Diego, CA), ML-7 (Calbiochem, San Diego, CA), latrunculin B (Calbiochem) were 40, 100, 100, 50, and 10-20 $\mu \mathrm{M}$, respectively. For drug experiments, NRK cells at prometaphase were treated for at least $10 \mathrm{~min}$ before anaphase onset and the drug was maintained during the following period of imaging. C3 transferase (Calbiochem) was microinjected at a concentration of $0.5 \mathrm{mg} / \mathrm{ml}$ with fluorescein dextran as a marker (Molecular Probes, Eugene, OR). Injection of fluorescein dextran alone had no effect on mitosis or cytokinesis.

\section{Immunofluorescence}

For myosin II staining, cells were fixed in 1\% formaldehyde, $0.1 \%$ glutaraldehyde, and $0.3 \%$ Triton X-100 for $1 \mathrm{~min}$, followed by postfixation in $0.5 \%$ glutaraldehyde for $10 \mathrm{~min}$. Myosin IIA was immunostained with anti-nonmuscle myosin IIA polyclonal antibodies (Covance Research, Princeton, NJ) at a dilution of $1: 100$. To stain for actin filaments, cells were fixed in $4 \%$ formaldehyde and $0.2 \%$ Triton X-100 for $10 \mathrm{~min}$ and incubated with Alexa488-phalloidin or Alexa-546-phalloidin following the manufacturer's protocol (Molecular Probes)

\section{Microscopy and Data Collection}

Images were collected with either a Zeiss Axiovert-10 or Axiovert-200M inverted microscope (Thornwood, NY), equipped with a $100 \times$, NA 1.30 Phase Plan-Neofluar objective lens for conventional epifluorescence optics, and a $100 \times$, NA $1.45 \alpha$ Plan-Fluar objective lens for TIRF-M. Light for TIRF-M was generated by a Lexel Model 94, $2 \mathrm{~W}$ argon ion laser operating at $10 \mathrm{~mW}, 488$ $\mathrm{nm}$ for GFP proteins, or a $2.5 \mathrm{~mW} 543 \mathrm{~nm}$ HeNe laser for mCherry-actin (model LHGR-0200, Research Electro Optics, Boulder, CO). The laser beam was expanded with a beam expander and directed into the microscope using a focusing lens and several steering mirrors, adjusted such that the beam exits the objective lens as a parallel beam at an angle exceeding the critical angle for total internal reflection. Fluorescence images were collected with a cooled CCD camera (model NTE/CCD-512-EBFT, Princeton Instrument, Trenton, NJ, or Model DV-887-DCS-BV, Andor Technology, Belfast, United Kingdom), with 0.5 - or 1-s exposure and subtracted with a dark-count image. Except for linear adjustments across the entire image to optimize the display, the images were unaltered. Data acquisition and analyses were performed with a combination of custom software, ImageJ (NIH; http://rsb.info.nih.gov/ij/) and Excel (Microsoft, Redmond, WA)

\section{Image Analysis}

Standard image analysis procedures, including kymograph analysis and intensity integration, were performed with a combination of custom software, ImageJ (NIH) and Excel (Microsoft). STICS was implemented as custom software, using a similar cross-correlation approach as described previously (Hebert et al., 2005; Ji and Danuser, 2005). Each set of displacement vectors was generated by averaging displacements detected in a set of five consecutive frames taken over $\sim 12 \mathrm{~s}$. The detection window for movements was set at $1.3 \times 1.3 \mu \mathrm{m}^{2}$ as the default and adjusted adaptively as described by Ji and Danuser (2005). TDM was also implemented as custom software. It involved pixelwise computation of the intensity differential, $\left(I_{t+\Delta t}-I_{t}\right) /\left(I_{t+\Delta t}+I_{t}\right) /$ $\Delta \mathrm{t} / 2$, where $I_{t}$ is the average intensity around the pixel at time $t$ and $\Delta \mathrm{t}=4-7 \mathrm{~s}$. This quantity equals approximately the local rate of percentage change in intensity. To control the noise some images were filtered with a median filter before the computation. For visualization, the resulting positive or negative values were scaled linearly and rendered in pseudocolor.
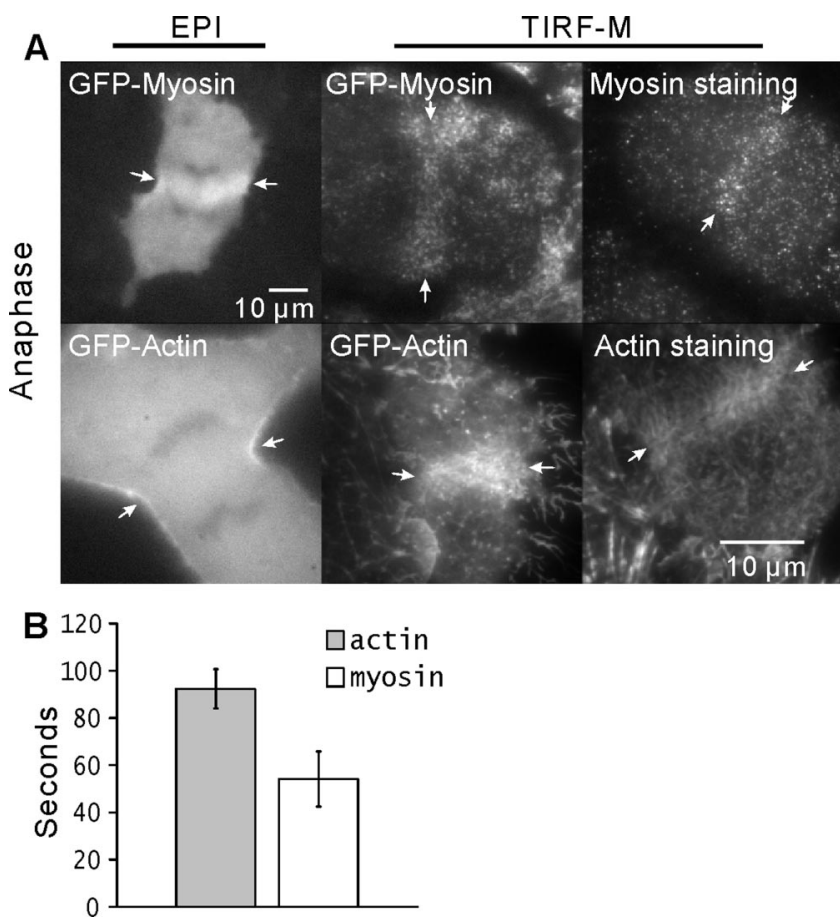

Figure 1. High-resolution imaging of cortical myosin and actin structure in mitotic cells with TIRF-M. GFP-nonmuscle myosin IIA heavy chain or GFP-actin accumulates along the equator, which is detected as a fuzzy band using conventional epifluorescence optics (A, left panels, arrows indicate the equator). TIRF-M provides a much higher resolution than epifluorescence, revealing fine cortical structures of myosin and actin (A, middle and right panels). Staining of endogenous myosin and actin (A, right panels) shows similar structures as those seen with GFP-myosin and GFP-actin in live cells (A, middle panels). GFP-myosin band appears in the equator slightly earlier than GFP-actin (B). Time (s) is measured from chromosome segregation to the first detection of GFP-myosin or GFPactin concentration along the equator $(\mathrm{n}=10$ each for actin and myosin; error bars, $\mathrm{SD}$ ).

\section{RESULTS}

\section{TIRF-M Provides High-Resolution Images of Cortical Structure of Myosin and Actin in Cytokinetic Cells}

To visualize myosin and actin in live cells, we expressed GFP-tagged nonmuscle myosin II heavy chain (referred as GFP-myosin later), or GFP- or mCherry-tagged actin in NRK epithelial cells, which remained relatively flat during mitosis and were ideal for microscopy. These tagged proteins served as reliable probes for endogenous structures (Supplementary Figure 1 and Figure 1A). Compared with conventional epifluorescence optics, TIRF-M provided a much higher resolution of cortical structures in mitotic cells (Figure 1A). GFP-myosin was found in dot-like structures with an average area of $0.2 \mu \mathrm{m}^{2}$ (or an equivalent diameter of $0.5 \mu \mathrm{m}$, which may be partially diffraction-limited), throughout the cortex of mitotic cells. From the lack of abrupt bleaching, these dots likely represent myosin minifilaments or small clusters of minifilaments as described previously (Verkhovsky and Borisy, 1993; Maupin et al., 1994), rather than single molecules. Cortical actin on dividing cells consisted of dots, patches, short fibers, and diffuse structures (Figure 1A).

Soon after anaphase onset, prominent myosin dots and actin filament structures appeared on the equatorial cortex, forming a prominent band before the initiation of ingression (Figure 1A). GFP-myosin band appeared along the equator 
slightly earlier than GFP-actin, becoming detectable at $\sim 60$ and $100 \mathrm{~s}$ after chromosome segregation, respectively (Figure 1B), suggesting that they may follow different pathways.

\section{Myosin Shows No Detectable Cortical Flow in Early Cytokinesis}

To determine if the assembly process of equatorial myosin involves cortical flow, as has been suggested previously (White and Borisy, 1983), we collected time-lapse TIRF-M movies of dividing cells expressing GFP-myosin. Direct observations of cortical myosin in more than 20 cells provided no indication of directional movement toward the equator during early equatorial assembly (Figure 2A, Supplemen- tary Video 1). Moreover, kymograph analysis showed only horizontal tracks indicating that there was no long-range movement of myosin throughout the cortex along any directions (Figure 2B). Consistent results were obtained with STICS, which tracked the movement of features within $1.3 \times$ $1.3 \mu \mathrm{m}^{2}$ regions (Figure $2 \mathrm{C}$ ). The increase in equatorial myosin concentration appeared to involve a combination of an increase in the intensity of pre-existing myosin structures and de novo appearance of new myosin dots along the equator (Figure $2 \mathrm{~A}$ and $2 \mathrm{~B} \mathrm{~d}$, e, boxed areas), which together lead to a $45 \pm 12 \%$ increase in average intensity along the equator during a 30-s interval in early cytokinesis. The flanking regions showed a concomitant intensity decrease of $14 \pm$
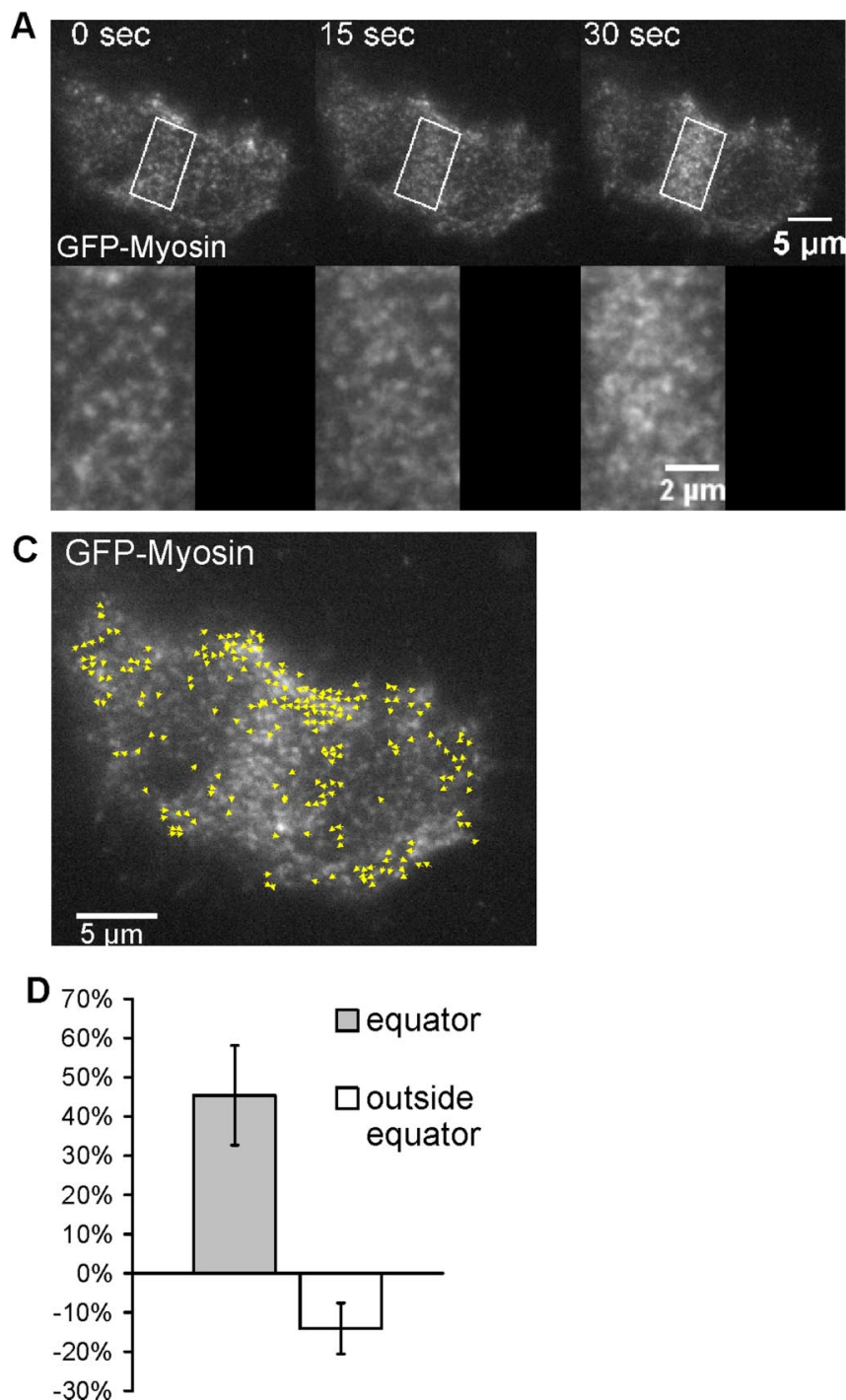

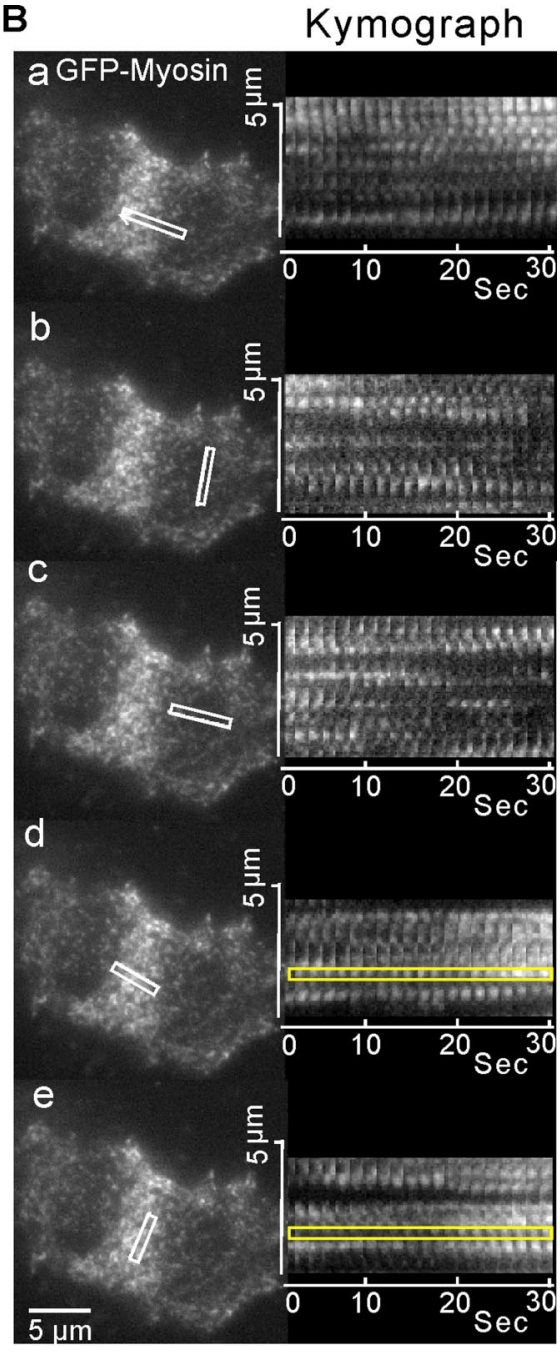

Figure 2. Lack of detectable cortical flow of myosin during early cytokinesis. Selected frames from a TIRF-M time-lapse sequence of cells expressing GFP-myosin show the rapid recruitment of myosin onto the equatorial cortex over a period of $30 \mathrm{~s}$ (A, top panels; bottom panels are the enlarged view of the equatorial region). Kymograph analysis of cortical myosin in different regions and along different directions (B, white rectangular boxes, left panels) shows no directed movement of cortical myosin dots during early assembly of the equatorial cortex (B, right panels). However, myosin dots in the equatorial region were more stable, as indicated by longer horizontal lines in kymographs (B, right panels). In addition, some myosin dots in the equatorial region gain intensity, whereas other dots appear to form de novo (B, boxes in kymographs of $\mathrm{d}$ and e). Analysis with STICS shows no significant coordinated movements of structures within detection windows of $1.3 \times$ $1.3 \mu \mathrm{m}^{2}$ throughout the cortex (C; yellow arrows indicate displacement velocity, cutoff velocity $=6 \mathrm{~nm} / \mathrm{s}$ ). The scale bar in C also represents a speed of $0.22 \mu \mathrm{m} / \mathrm{s}$ in STICS. (D) Analysis of integrated fluorescence intensities over a period of $30 \mathrm{~s}$ during early cytokinesis indicates an increase of average intensity by $45 \%$ along the equator and a decrease by $14 \%$ in the flanking region $(n=5$, error bars, SD). Supplementary Video 1 shows the corresponding movie. 

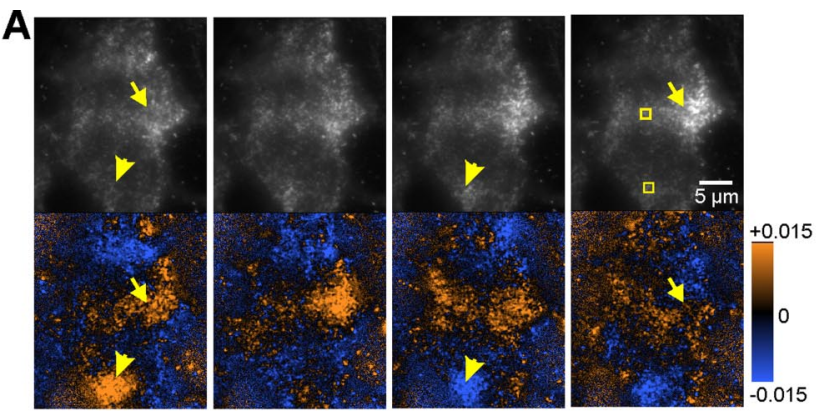

Figure 3. Random domains of myosin as-
sembly/association and inhibition of myosin disassembly/dissociation along the equatorial cortex. (A) TDM reveals highly dynamic domains of myosin assembly/association and disassembly/dissociation throughout the cortex during early cytokinesis. Regions of assembly/association are shown in orange and regions of disassembly/dissociation shown in blue. (B) During the assembly of the equatorial band, disassembly is suppressed along the equator, such that strong increases in intensity along the equator are followed by weak increases or steady intensities (A, arrows, and B), whereas strong increases outside the equator are followed by strong decreases (A, arrowheads, and $\mathrm{C})$. Yellow boxes in $\mathrm{A}$ indicate the regions plotted (green curves in B and C). Green, red, and blue curves represent the rate of intensity change and the average intensity in equatorial or polar regions of $20 \times 20$ pixels in three individual cells ( $B$ and $C)$. The average intensity at $t=0$ is set as 100 arbitrary units. Supplementary Video 2 shows the corresponding movie.
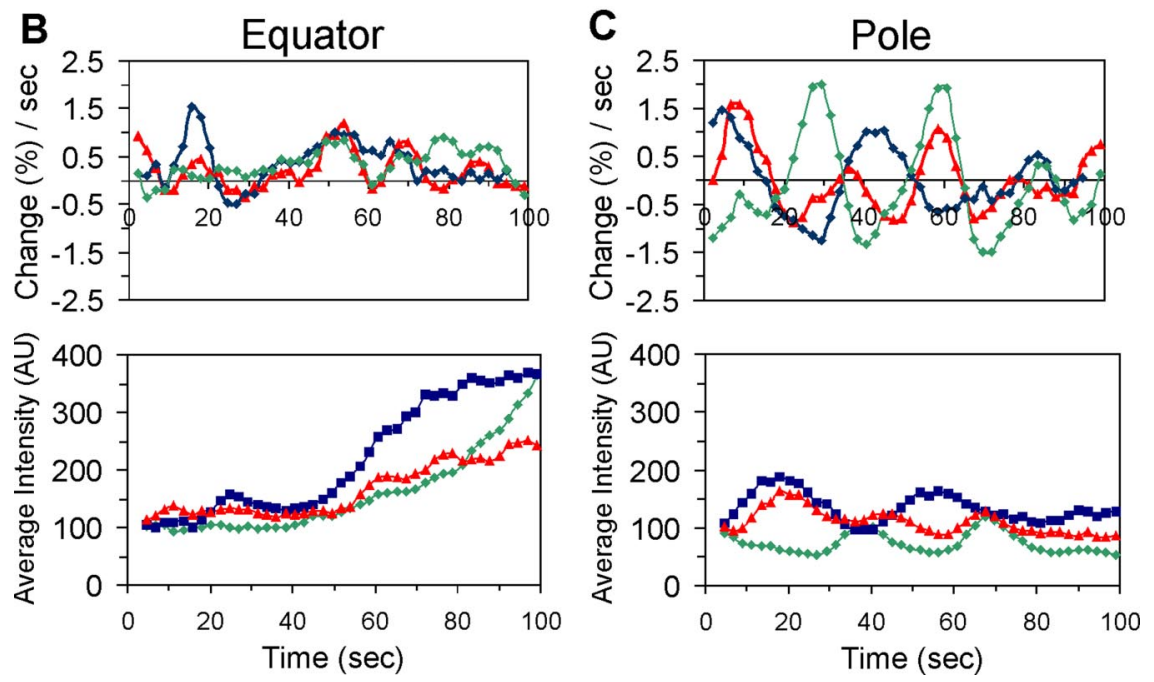

$6 \%$ (Figure 2D), suggesting changes in the relative association and dissociation kinetics between the equatorial region and the rest of the cortex.

\section{Cortical Myosin Assembly/Association Appears in Dynamic Domains throughout the Cortex}

TIRF-M time-lapse recording of cortical myosin revealed an intriguing phenomenon. Contrary to the common notion that myosin builds up directly and locally along the equator, we observed domains over the entire cell cortex where myosin intensity showed transient increases (Figure 3A, and Supplementary Video 2). No such domains were observed with cells expressing GFP-actin or GFP alone. To visualize more directly myosin intensity dynamics and to normalize against the local concentration, we developed TDM, which calculates the rate of temporal differential, $\left(\mathrm{I}_{\mathrm{t}+\Delta t}-\mathrm{I}_{\mathrm{t}}\right) /\left(\mathrm{I}_{\mathrm{t}+\Delta t}+\right.$ $\left.\mathrm{I}_{\mathrm{t}}\right) / \Delta \mathrm{t} / 2$, with pairs of images $\mathrm{I}_{\mathrm{t}}, \mathrm{I}_{\mathrm{t}+\Delta \mathrm{t}}$ separated temporally by $\Delta t$. This quantity approximates the rate of percentage change in intensity. The resulting images were rendered in color, with bright orange marking regions of strong proportional increases in intensity and bright blue marking regions of strong decrease ( $\mathrm{n}=31$; Figure $3 \mathrm{~A}$, and Supplementary Video 2). TDM revealed strong assembly domains of myosin up to $10 \mu \mathrm{m}$ in diameter throughout the cortex. These domains lasted typically 15-20 s, during which some of them traveled across the cortex (Figure 3A). These observations indicate that myosin assembly was not confined to the equatorial region (Lucero et al., 2006), although the equatorial region may have a higher net assembly activity than elsewhere.

TDM also revealed suppression of disassembly/dissociation activities along the equator. Although a strong phase of assembly outside the equator was typically followed by an equally strong phase of disassembly, assembly along the equator was followed by a neutral phase (Figure 3B, and Supplementary Video 2), when the intensity remained steady or increased slowly. Therefore, a net increase in equatorial myosin may be achieved through a rectifier-like effect, by allowing assembly or association on the entire cortex while suppressing disassembly or dissociation along the equator (Figure 3B). The regulation of myosin disassembly or dissociation may thus play a crucial role in equatorial recruitment.

\section{Cortical Myosin Is Affected in Different Ways by Small GTPase Rho, Rho Kinase, Myosin Light-Chain Kinase, and Its Own ATPase Activities during Cytokinesis}

Cortical dynamics of myosin is likely affected by its ATPase and self-assembly activities, which are in turn regulated by the phosphorylation of myosin regulatory light chain (MRLC) by a number of kinases including the myosin lightchain kinase (MLCK) and Rho-dependent kinase (ROK; Bresnick, 1999; Poperechnaya et al., 2000; Fukata et al., 2001; Chew et al., 2002; Matsumura, 2005). Y-27632, an inhibitor of $\mathrm{ROK}$, delayed cytokinesis as reported previously (Kosako et al., 2000). The formation of equatorial myosin band appeared uninhibited (Figure 4A). However, time-lapse movies and kymographs indicated that equatorial myosin dots were surprisingly stable in Y-27532-treated cells (Figure 4A). Myosin dots outside the equator became sparse and highly transient compare with those in control cells shown in Figure 2, suggesting that they failed to associate stably with the cortex (Figure 4A; Supplementary Video 3). In addition, TDM analysis showed a strong inhibition of dynamic myosin domains throughout the cortex (Figure 4D). 

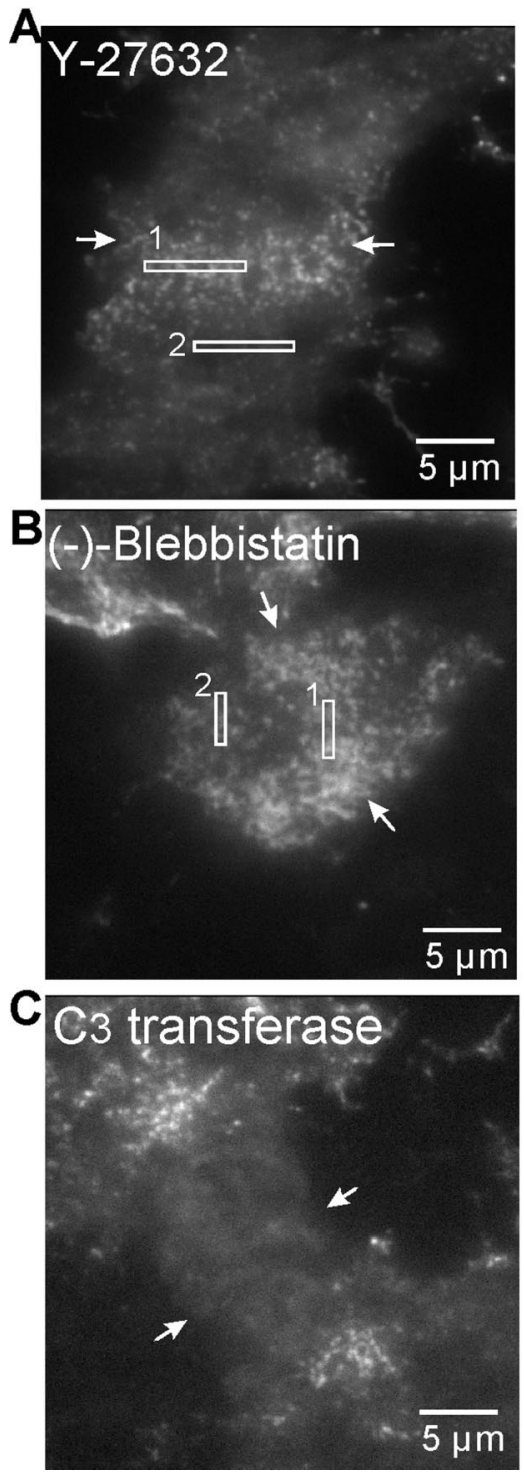

Kymograph
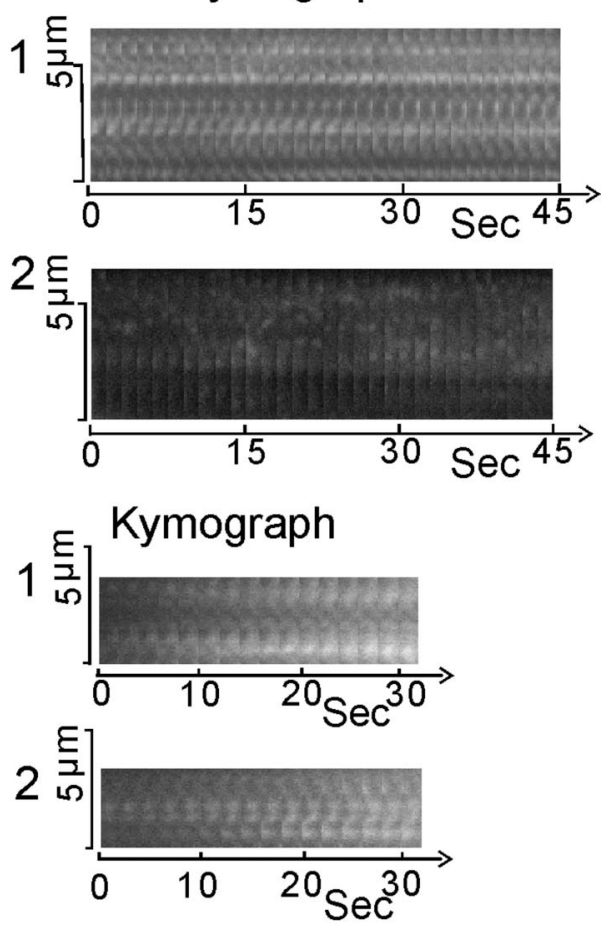

D

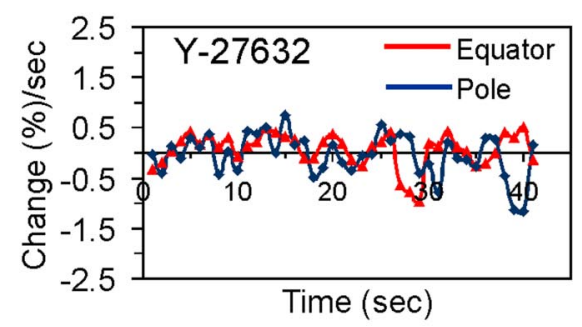

Figure 4. Different effects of inhibitors of ROK, Rho, and myosin ATPase on cortical myosin dynamics during cytokinesis. Myosin localization along the equator appears uninhibited in a cell treated with Y-27632, a ROK inhibitor (A, left panel, arrows indicate equator). Kymograph analysis indicates that myosin dots in the equatorial region become surprisingly stable (A, kymograph 1, right top panel), whereas dots away from the equator become weak and highly unstable (A, kymograph 2, right bottom panel) compared with those in similar regions of control cells (Figure $2 \mathrm{~B}, \mathrm{~b}$ and e). Supplementary Video 3 shows the corresponding movie. Injection of C3 transferase, a Rho GTPase inhibitor, in contrast, causes a strong inhibition of myosin localization along the equator $(\mathrm{C}$, arrows indicate equator). (-)-Blebbistatin, a myosin ATPase inhibitor, increases cortical myosin intensity both inside and outside the equatorial region (B, arrows indicate equator). Supplementary Video 4 shows the corresponding movie. TDM analysis shows the strong inhibition of intensity fluctuations by Y-27632 (D), in contrast to the waves seen in control cells (Figure 3, B and C).
Injection of C3 transferase (a Rho inhibitor), unlike Y-27632 treatment, caused complete inhibition of the recruitment of myosin dots along the equator (Figure 4C, Supplementary Figure 2; (O'Connell et al., 1999; Kamijo et al., 2006), suggesting that ROK is not the only effector of Rho that regulates equatorial myosin recruitment. ML-7, an inhibitor of MLCK, caused a delay in cytokinesis without an apparent effect on cortical myosin dots along or outside the equator (Supplementary Video 4).

Because C3 and Y-27632 presumably inhibit myosin ATPase by inhibiting MRLC phosphorylation, we compared their effects with the effect of $(-)$-blebbistatin, a potent myosin ATPase inhibitor. Blebbistatin caused an increase in the intensity of myosin dots throughout the cortex (Figure 4B, Supplementary Video 5), by $97 \%$ along the equator and $71 \%$ outside the equator during a 30-s period in early cytokinesis. The different effects between (-)-blebbistatin and Rho/ROK inhibitors suggest that myosin ATPase activity and MRLC phosphorylation play distinct roles in cortical myosin recruitment. Also relevant was the previous demonstration that equatorial myosin recruitment took place in yeast, Drosophila and HeLa cells treated with latrunculin B (Wu et al.,
2003; Dean et al., 2005; Kamijo et al., 2006), which induces depolymerization of actin filaments and thereby inhibits the actomyosin ATPase. This was also the case for NRK cells (Supplementary Figure 3, and Supplementary Video 6). However, the equatorial myosin band gradually widened over $\sim 20$ min after anaphase onset, suggesting that acto-myosin interactions play a role in restricting the equatorial myosin localization (Supplementary Figure 3).

\section{Recruitment of Equatorial Actin Involves a Combination of Myosin Motor-dependent Fluxes and De Novo Assembly}

The delay of equatorial actin recruitment relative to that of myosin suggested that actin and myosin may follow different pathways (Figure 1B). To observe directly the equatorial recruitment of actin, we collected time-lapse movies of dividing cells expressing GFP-actin or mCherry-actin. In contrast to myosin, actin filaments showed a striking flux toward the equator during cytokinesis (observed in 20 cells; Figure 5, A and B, Supplementary Videos 7 and 8), at an estimated average speed of $2.1 \pm 0.6 \mu \mathrm{m} / \mathrm{min}$ based on the slopes of kymographs (Figure 6B). The flux was unlikely to 

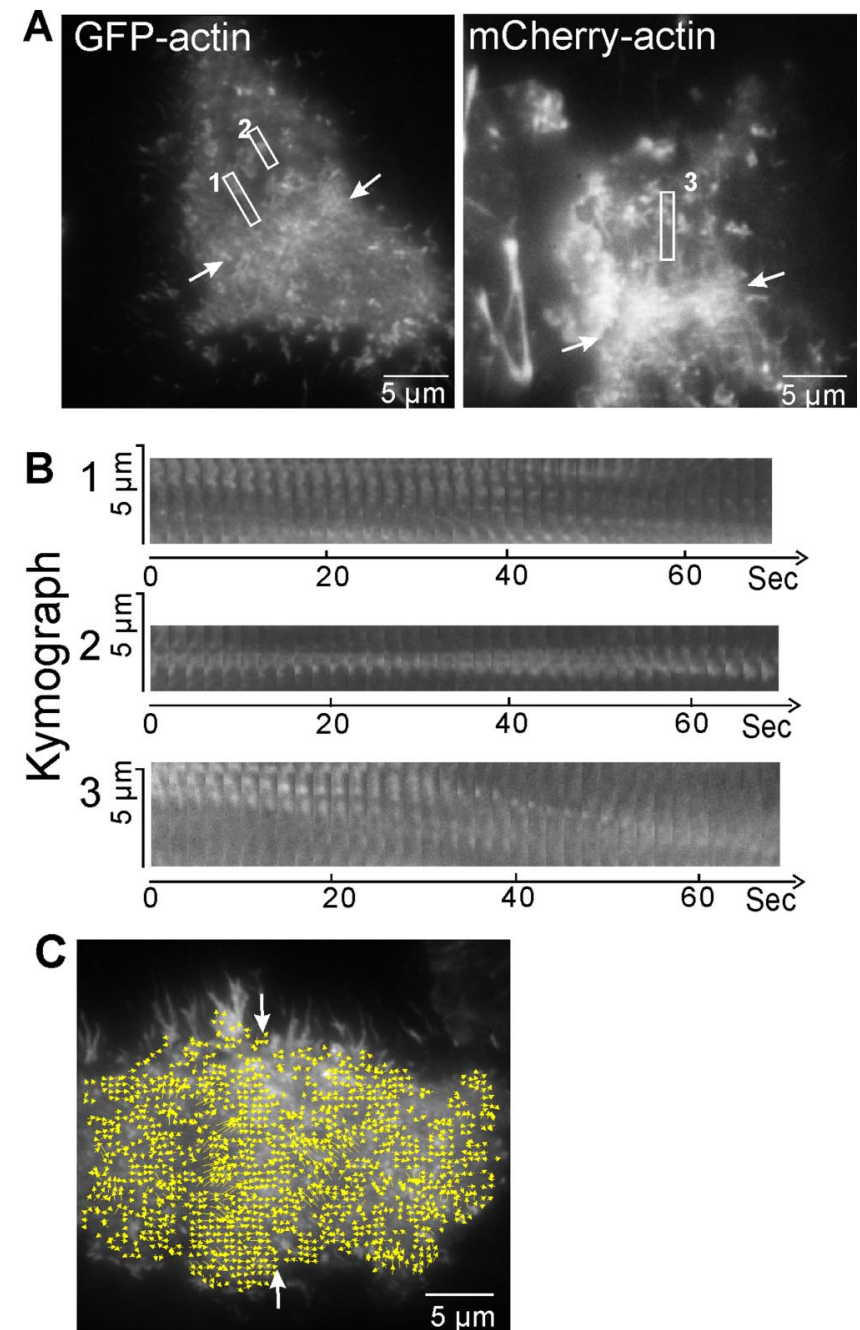

Figure 5. Striking actin flux toward the equator during early cytokinesis. Cells expressing GFP-actin or mCherry-actin show striking actin flux toward the equator during early cytokinesis (A, Supplementary Videos 7 and 8). White rectangular boxes indicate the regions analyzed in kymographs, and arrows indicate the equator (A). Kymograph analysis (B) and STICS analysis (C) show some actin structures on the cortex move toward equator $(B$, panels 1 and $3)$, whereas others do not move (B, panel 2). Thus the flux does not represent global cortical movement. The scale bar also represents a speed of $0.22 \mu \mathrm{m} / \mathrm{s}$ in STICS (C).

reflect the movement of the entire cortical actin structure, because some actin structures moved to the equator, whereas others remained stationary (Figure 5B). In addition, small actin structures were observed to dissociate from large patches and move into the furrow, suggesting that actin disassembly or severing, rather than a global collapse of the cortical network, may be involved in the generation of the flux (Supplementary Video 7). Moreover, STICS analysis indicated heterogeneous speeds and movement patterns, and juxtaposition of directional and random movements (Figure 5C), further arguing against global movement of a cross-linked cortex.

The long-range, directed movement of actin filaments implied the involvement of motor-dependent forces. To determine if myosin motor activities were responsible for the actin flux, we investigated the effect of $(-)$-blebbistatin. The treatment with (-)-blebbistatin immediately inhibited the actin flux, whereas $(+)$-blebbistatin (an inactive form of blebbistatin as negative control) had no effect (Figure 6A; Supplementary Video 9). The slopes in the kymographs indicate an average flux speed of $2.1 \mu \mathrm{m} / \mathrm{min}$ in control cells and close to zero in (-)-blebbistatin treated cells (Figure 6B), suggesting that myosin motor activities were critical for the actin flux. However, equatorial concentration of actin filaments took place despite the inhibition of actin flux (Supplementary Videos 9 and 10). TDM further confirmed the persistence of actin assembly activities along the equator in the presence of (-)-blebbistatin (Figure 7). Therefore both filament flux and de novo assembly were involved in actin recruitment along the equator.

\section{DISCUSSION}

Equatorial concentration of actin and myosin represents a common feature of cytokinesis seen from yeast to mammalian cells. Despite the variability in the extent of concentration, likely due to the feedback from cortical resistance to ingression and the balance between assembly and disassembly activities (Fishkind and Wang, 1995), it is generally believed that the recruitment process holds an important key to the understanding of the mechanism of cytokinesis. For example, the polar relaxation hypothesis predicts that the entire cortical structures should move toward the equator, due to the weak forces in the polar region relative to the strong contractions along the equator (White and Borisy, 1983). In contrast, some versions of the equatorial contraction mechanism emphasize a de novo mechanism for equatorial structural assembly (Wu et al., 2006), whereas other versions incorporate cortical flow as part of an assembly process (He and Dembo, 1997). However, because of the lack of high-resolution images depicting the recruitment process in mammalian cells, there has been no definitive evidence for these proposed mechanisms. The clarity of images provided by TIRF-M has allowed us to determine unambiguously the behavior of cortical actin and myosin during early cytokinesis.

\section{Equatorial Recruitment of Myosin in Early Cytokinesis Does Not Involve Cortical Flow}

Although dot-like structures of cortical myosin have been observed during cytokinesis in fixed samples by confocal microscope (Maupin et al., 1994), little is known about how they become concentrated along the equator. Our results, showing no detectable, long-range flow of myosin dots toward the equator, argued against the cortical flow hypothesis for myosin recruitment and supported either the assembly of myosin minifilaments from cytoplasmic subunits or recruitment of preassembled minifilaments. Although the polar cortex showed a net loss of myosin dots, myosin appeared to be released into cytoplasm first and recruited to the equator. This shift in the balance of myosin cortical association may involve differential interactions of polar and equatorial cortices with astral microtubules (Werner et al., 2007).

Our results appear to contradict previously reported fluxes of myosin in mammalian cells (DeBiasio et al., 1996), and in Dictyostelium (Yumura, 2001). However, the present observations were focused on early assembly events of equatorial cortex. During the subsequent phase of active ingression, we did observe some limited movements of myosin dots on the equatorial cortex and in the immediately adjacent region, which likely reflect contractile activities and may explain previous observations of myosin movements (DeBiasio et al., 1996). In addition, different organisms may adopt different mechanisms of myosin recruitment, as sug- 

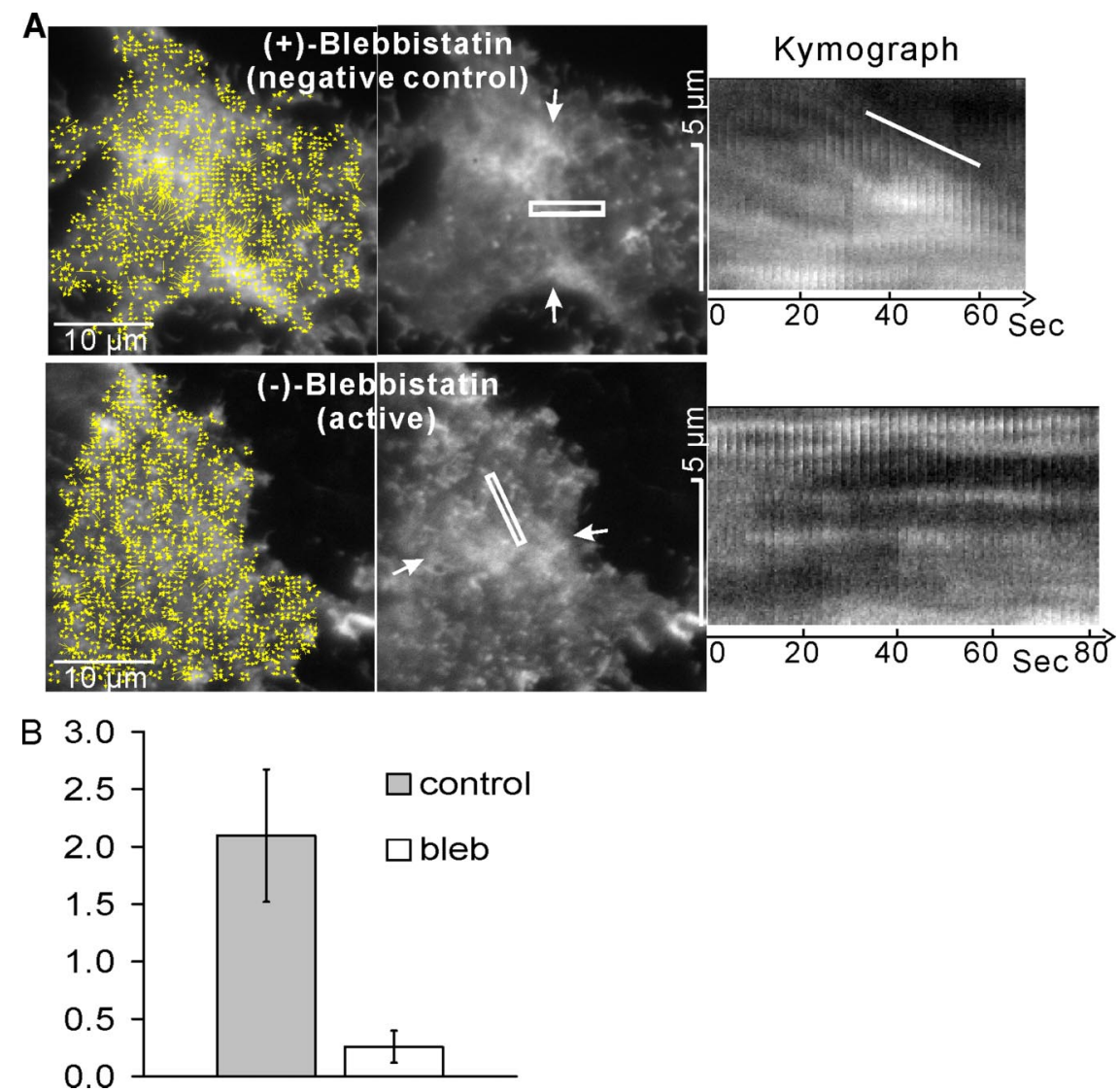

Figure 6. Requirement of myosin motor activity for the actin flux. Treatment with active (-)-blebbistatin abolishes actin flux (A, bottom panel), whereas treatment with the inactive isomer (+)-blebbistatin has no effect (A, top panel), as suggested by both STICS (A, left panels) and kymographs (A, right panels). White rectangular boxes indicate the regions analyzed by kymographs and arrows indicate the equator (A, middle panel, and Supplementary Video 9). The slopes in the kymographs indicate an average flux speed of 2.1 $\mu \mathrm{m} / \mathrm{min}$ in control cells and close to zero in (-)-blebbistatin-treated cells (B; $\mathrm{n}=19$ for the control and $\mathrm{n}=8$ for blebbistatin; error bars, $\mathrm{SD})$.

gested by the multiple mechanisms of cytokinesis in motile Dictyostelium cells (Uyeda et al., 2004).

\section{Equatorial Recruitment of Actin Involves a Combination of Cortical Flow and De Novo Assembly}

In contrast to myosin, cortical actin showed a striking flux toward the equator, consistent with previous observations at a limited resolution using conventional optics and microinjected fluorescent phalloidin (Cao and Wang, 1990). In ad- dition, the flux took place in discrete domains flanking the equator, and at least part of the moving structures appeared to be generated by severing and/or disassembling existing actin structures, suggesting that the flux was an active, selective process rather than global cortex movement as a result of force balance.

Treatment of blebbistatin abolished the actin flux and inhibited actin turnover, but did not inhibit actin concentration in the equator (Figure 7; Guha et al., 2005; Murthy and
A
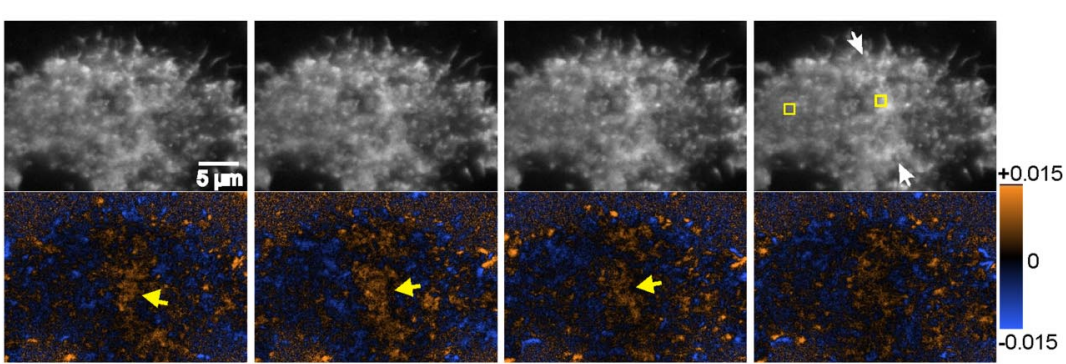

B
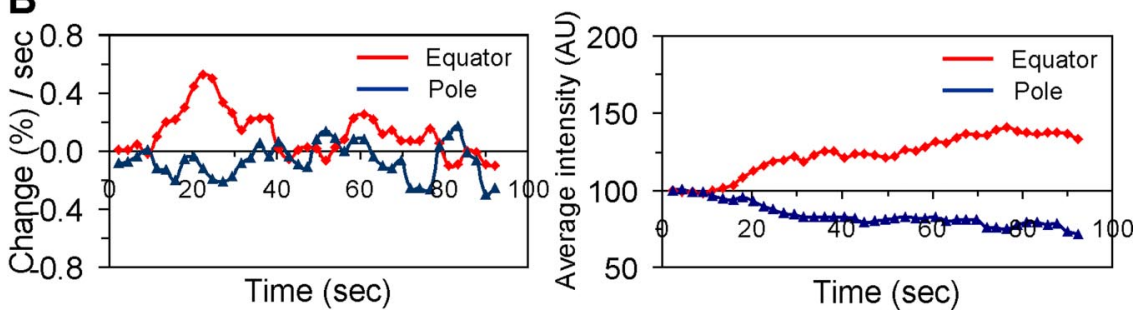

Figure 7. Involvement of both myosin motor-dependent fluxes and de novo assembly in the recruitment of equatorial actin. TDM of cell treated with active (-)-blebbistatin shows inhibition of actin flux and cytokinesis, whereas the equatorial region shows net assembly (A, arrows indicate equator). Plots of percentage intensity change per second (B, left panel) or average intensity in regions of $20 \times 20$ pixels (B, right panel) show a broad peak of assembly activities along the equator during early cytokinesis (B, red curve), whereas the polar region shows fluctuating values around the baseline ( $\mathrm{B}$, blue curve). The regions plotted are indicated by yellow boxes in the corresponding TIRF-M image (A, right panel). The average intensity at $t=0$ is set as 100 arbitrary units. Supplementary Video 10 shows the corresponding movie. 
Wadsworth, 2005), suggesting that the flux was driven by myosin motor activities and that there were additional, fluxindependent mechanisms, likely de novo assembly as suggested in other systems (Noguchi and Mabuchi, 2001; Pelham and Chang, 2002; Severson et al., 2002; Wu et al., 2006). Myosin may be involved in both processes, by providing forces for the flux and by facilitating the turnover of actin cortex to generate the sources of flux. Because blebbistatin also completely inhibited furrow ingression, it is possible that cytokinesis requires not only an equatorial acto-myosin band but also actin flux and/or turnover. In addition, although the major events of cytokinesis take place along the equator, the complex picture presented by TIRF-M suggested that the process may involve the entire cortex.

\section{Equatorial Myosin Recruitment Involves the Regulation of Both Assembly and Disassembly Processes}

Dynamic cytoskeletal structures are maintained by a balance between assembly and disassembly activities. Previous immunostaining studies have shown a concentration of monoand di-phosphorylated MRLC along the equator, which is known to activate the myosin ATPase and promote the assembly of filamentous myosin structures (Matsumura et al., 1998, 2001;). These observations were often interpreted as suggesting an increase in the rate of myosin assembly or cortical association along the equator. However, equatorial MRLC phosphorylation and myosin structures, may be stimulated equally effectively by decreasing the rate of disassembly or dissociation from the equatorial cortex, as was suggested by the requirement of regulated myosin phosphatase activities in cytokinesis (Matsumura, 2005).

Time-lapse TIRF-M and TDM revealed surprisingly active domains of cortical myosin assembly/association throughout the cell cortex immediately after anaphase onset. These domains were not detected in previous studies, because without TIRF-M, their contrast against the strong cytoplasmic signals was likely to be very low. In addition, the transient, random nature of these domains likely made them very difficult to notice in fixed cells. The global presence of these assembly domains suggested that assembly signals for myosin were not confined to the equatorial region (Lucero et al., 2006).

Previous studies showed that the primary function of ROK during cytokines is to elevate the phosphorylation of MRLC (Dean and Spudich, 2006). The strong inhibition of dynamic domains of cortical myosin assembly by Y-27632, as shown in the present study, suggested that ROK and ROK-mediated phosphorylation of MRLC were involved in the recruitment of myosin throughout the cortex. However, the presence of equatorial myosin band despite the inhibition of ROK suggested a second, ROK-independent pathway that directly recruited myosin to the equatorial cortex.

TDM analysis further revealed local inhibition of myosin disassembly/dissociation along the equator, which likely contributed to the build up of myosin concentration. Unexpectedly, the stability of equatorial myosin in the presence of Y-27632 suggested that the stabilization involved a mechanism independent of ROK-induced direct MRLC phosphorylation or inhibition of myosin phosphatase (Fukata et al., 2001), whereas the turnover was dependent on ROK. Furthermore, actin filaments may play a role in the stability of myosin, as suggested by the gradual expansion of the equatorial myosin band in latrunculin-treated cells. Other molecules such as anillin have also been implicated in the recruitment of myosin, possibly by stabilizing myosin association along the equator (Straight et al., 2005).
The effects of blebbistatin on cortical myosin distribution and dynamics were strikingly different from those of Y-27632 or C3. Inhibition of myosin ATPase activity by blebbistatin appeared to inhibit cortical myosin disassembly/dissociation without affecting cortical myosin assembly/association, indicating that the ATPase activity is not required for its cortical association but may be required for its turnover. In contrast, C3caused strong inhibition of equatorial myosin recruitment, suggesting that the primary effect of Rho on myosin was not the regulation of ATPase activities, but possibly its self assembly, disassembly, and/or cortical association.

In conclusion, many previous models of cortical ingression involved global, coupled movements of actin and myosin filaments into the equatorial region. The present study showed that, while a prominent myosin-dependent actin flux did occur during the cytokinesis of NRK cells, the movement was by no means global and no such movement was detectable for myosin. In addition, the formation of the equatorial actin band involved a second, myosin-independent, de novo assembly process. These results support an active mechanism that promotes structural organization at the equator, rather than a passive mechanism as a consequence of differential cortical contractile activities. Moreover, the discovery of dynamic domains of myosin assembly throughout the cortex, combined with a localized suppression of myosin disassembly/dissociation along the equator, suggests that the spatial and temporal control of cytokinesis may involve the regulation of both assembly and disassembly activities.

\section{ACKNOWLEDGMENTS}

The authors thank Dr. Michael Davidson (Florida State University) for sharing the mCherry-actin plasmids, and Dr. Robert Adelstein (NIH/NHLBI) for the GFP-myosin II plasmids. This study was supported by Grant GM-32476 from the National Institutes of Health to Y.L.W.

\section{REFERENCES}

Axelrod, D. (2001). Total internal reflection fluorescence microscopy in cell biology. Traffic 2, 764-774.

Bray, D., and White, J. G. (1988). Cortical flow in animal-cells. Science 239, $883-888$.

Bresnick, A. R. (1999). Molecular mechanisms of nonmuscle myosin-II regulation. Curr. Opin. Cell Biol. 11, 26-33.

Burgess, D. R., and Chang, F. (2005). Site selection for the cleavage furrow at cytokinesis. Trends Cell Biol. 15, 156-162.

Cao, L. G., and Wang, Y. L. (1990). Mechanism of the formation of contractile ring in dividing cultured animal cells. II. Cortical movement of microinjected actin filaments. J. Cell Biol. 111, 1905-1911.

Chew, T. L., Wolf, W. A., Gallagher, P. J., Matsumura, F., and Chisholm, R. L. (2002). A fluorescent resonant energy transfer-based biosensor reveals transient and regional myosin light chain kinase activation in lamella and cleavage furrows. J. Cell Biol. 156, 543-553.

Dean, S. O., Rogers, S. L., Stuurman, N., Vale, R. D., and Spudich, J. A. (2005) Distinct pathways control recruitment and maintenance of myosin II at the cleavage furrow during cytokinesis. Proc. Natl. Acad. Sci. USA 102, 1347313478 .

Dean, S. O., and Spudich, J. A. (2006). Rho kinase's role in myosin recruitment to the equatorial cortex of mitotic Drosophila S2 cells is for myosin regulatory light chain phosphorylation. PLoS ONE 1, e131.

DeBiasio, R. L., LaRocca, G. M., Post, P. L., and Taylor, D. L. (1996). Myosin II transport, organization, and phosphorylation: evidence for cortical flow/ solation-contraction coupling during cytokinesis and cell locomotion. Mol. Biol. Cell 7, 1259-1282.

Fishkind, D. J., and Wang, Y. L. (1995). New horizons for cytokinesis. Curr Opin. Cell Biol. 7, 23-31. 
Fukata, Y., Amano, M., and Kaibuchi, K. (2001). Rho-Rho-kinase pathway in smooth muscle contraction and cytoskeletal reorganization of non-muscle cells. Trends Pharmacol. Sci. 22, 32-39.

Guha, M., Zhou, M., and Wang, Y. L. (2005). Cortical actin turnover during cytokinesis requires myosin II. Curr. Biol. 15, 732-736.

Gunsalus, K. C., Bonaccorsi, S., Williams, E., Verni, F., Gatti, M., and Goldberg, M. L. (1995). Mutations in twinstar, a Drosophila gene encoding a cofilin/ADF homologue, result in defects in centrosome migration and cytokinesis. J. Cell Biol. 131, 1243-1259.

He, X., and Dembo, M. (1997). On the mechanics of the first cleavage division of the sea urchin egg. Exp. Cell Res. 233, 252-273.

Hebert, B., Costantino, S., and Wiseman, P. W. (2005). Spatiotemporal image correlation spectroscopy (STICS) theory, verification, and application to protein velocity mapping in living $\mathrm{CHO}$ cells. Biophys. J. 88, 3601-3614.

Ji, L., and Danuser, G. (2005). Tracking quasi-stationary flow of weak fluorescent signals by adaptive multi-frame correlation. J, Microsc, 220, 150-167.

Kamijo, K., Ohara, N., Abe, M., Uchimura, T., Hosoya, H., Lee, J. S., and Miki, T. (2006). Dissecting the role of Rho-mediated signaling in contractile ring formation. Mol. Biol. Cell 17, 43-55.

Kosako, H., Yoshida, T., Matsumura, F., Ishizaki, T., Narumiya, S., and Inagaki, M. (2000). Rho-kinase/ROCK is involved in cytokinesis through the phosphorylation of myosin light chain and not ezrin/radixin/moesin proteins at the cleavage furrow. Oncogene 19, 6059-6064.

Lucero, A., Stack, C., Bresnick, A. R., and Shuster, C. B. (2006). A global, myosin light chain kinase-dependent increase in myosin II contractility accompanies the metaphase-anaphase transition in sea urchin eggs. Mol. Biol. Cell 17, 4093-4104.

Matsumura, F. (2005). Regulation of myosin II during cytokinesis in higher eukaryotes. Trends Cell Biol. 15, 371-377.

Matsumura, F., Ono, S., Yamakita, Y., Totsukawa, G., and Yamashiro, S. (1998). Specific localization of serine 19 phosphorylated myosin II during cell locomotion and mitosis of cultured cells. J. Cell Biol. 140, 119-129.

Matsumura, F., Totsukawa, G., Yamakita, Y., and Yamashiro, S. (2001). Role of myosin light chain phosphorylation in the regulation of cytokinesis. Cell Struct. Funct. 26, 639-644.

Maupin, P., Phillips, C. L., Adelstein, R. S., and Pollard, T. D. (1994). Differential localization of myosin-II isozymes in human cultured cells and blood cells. J. Cell Sci. 107(Pt 11), 3077-3090.

Murthy, K., and Wadsworth, P. (2005). Myosin-II-dependent localization and dynamics of F-actin during cytokinesis. Curr. Biol. 15, 724-731.
Noguchi, T., and Mabuchi, I. (2001). Reorganization of actin cytoskeleton at the growing end of the cleavage furrow of Xenopus egg during cytokinesis. J. Cell Sci. 114, 401-412.

O'Connell, C. B., Wheatley, S. P., Ahmed, S., and Wang, Y. L. (1999). The small GTP-binding protein rho regulates cortical activities in cultured cells during division. J. Cell Biol. 144, 305-313.

Pelham, R. J., and Chang, F. (2001). Actin polymerization occurs at the contractile ring and is required for efficient cytokinesis in S. pombe. Mol. Biol. Cell 12, 423a-423a.

Pelham, R. J., and Chang, F. (2002). Actin dynamics in the contractile ring during cytokinesis in fission yeast. Nature 419, 82-86.

Poperechnaya, A., Varlamova, O., Lin, P. J., Stull, J. T., and Bresnick, A. R. (2000). Localization and activity of myosin light chain kinase isoforms during the cell cycle. J. Cell Biol. 151, 697-708.

Severson, A. F., Baillie, D. L., and Bowerman, B. (2002). A Formin Homology protein and a profilin are required for cytokinesis and Arp2/3-independen assembly of cortical microfilaments in C. elegans. Curr. Biol. 12, 2066-2075.

Straight, A. F., Field, C. M., and Mitchison, T. J. (2005). Anillin binds nonmuscle myosin II and regulates the contractile ring. Mol. Biol. Cell 16, 193201.

Uyeda, T. Q., Nagasaki, A., and Yumura, S. (2004). Multiple parallelisms in animal cytokinesis. Int. Rev. Cytol. 240, 377-432.

Verkhovsky, A. B., and Borisy, G. G. (1993). Non-sarcomeric mode of myosin II organization in the fibroblast lamellum. J. Cell Biol. 123, 637-652.

Wang, Y. L. (2005). The mechanism of cortical ingression during early cytokinesis: thinking beyond the contractile ring hypothesis. Trends Cell Biol. 15, 581-588.

Wang, Y. L., Silverman, J. D., and Cao, L. G. (1994). Single particle tracking of surface receptor movement during cell division. J. Cell Biol. 127, 963-971.

Werner, M., Munro, E., and Glotzer, M. (2007). Astral signals spatially bias cortical Myosin recruitment to break symmetry and promote cytokinesis. Curr. Biol. 17, 1286-1297

White, J. G., and Borisy, G. G. (1983). On the mechanisms of cytokinesis in animal cells. J. Theor. Biol. 101, 289-316.

Wu, J. Q., Kuhn, J. R., Kovar, D. R., and Pollard, T. D. (2003). Spatial and temporal pathway for assembly and constriction of the contractile ring in fission yeast cytokinesis. Dev. Cell 5, 723-734.

Wu, J. Q., Sirotkin, V., Kovar, D. R., Lord, M., Beltzner, C. C., Kuhn, J. R., and Pollard, T. D. (2006). Assembly of the cytokinetic contractile ring from a broad band of nodes in fission yeast. J. Cell Biol. 174, 391-402.

Yumura, S. (2001). Myosin II dynamics and cortical flow during contractile ring formation in Dictyostelium cells. J. Cell Biol. 154, 137-146. 